\title{
Goal Oriented Value Object Classification for Healthcare Service Development
}

\author{
Gilmini Dantanarayana ${ }^{1}$, Chathurika Wickramage ${ }^{1}$, and Prasad Jayaweera ${ }^{2}$ \\ ${ }^{1}$ Department of Computer Science, Faculty of Science, University of Ruhuna, Sri Lanka \\ ${ }^{2}$ Department of Computer Science, Faculty of Applied Sciences, \\ University of Sri Jayewardenepura, Sri Lanka \\ \{gilmini, chathurika\}@dcs.ruh.ac.1k, pja@sjp.ac.lk
}

\begin{abstract}
In present day, performance of inter and intra cooperative enterprise systems can be guaranteed by provisioning e-Services. Modeling values for eService is challenging due to inherited complexity of service constellations. In this research, we present a classification to guide identification of different types of value objects that could be considered as mostly relevant and appropriate in healthcare trading scenarios. Further we propose a set of guidelines that direct construction of $\mathrm{e}^{3}$-Value model along with the instructions to figure out the value objects. The classification and the guidelines are capable enough to provide clear and precise understanding of goal aligned e-Services to be developed and deployed by e-Service designers. Thus the proposed approach also facilitates business/IT alignment by realizing business motivations and top level goals in $\mathrm{e}^{3}$-Value model which directly assist in defining business system requirements.
\end{abstract}

Keywords: Healthcare Services, Standards, Motivations \& Value Modeling.

\section{$1 \quad$ Introduction}

Nowadays most enterprise solution developments are based on service orientation and related modeling concepts in order to cope with demanding flexibility, portability and agility for successful and sustainable business service deployments. Especially requirements of healthcare domain can be modeled as services offering wide spectrum of services to its customer, the patient. A notable global standardization effort in this direction is SAIF [7]. However, these efforts still lacks complete and systematic guidelines or approaches that could facilitate healthcare service designing and deployment. This paper attempts to propose a service oriented Value Objects (VO) identification based on BMM and $\mathrm{e}^{3}$-Value constructs as part of an ongoing research for a complete framework for health solution development in line with global standardizations efforts introduced briefly in the next section of the paper.

The composition of concepts used, actions performed and relationships among users in an enterprise should be represented and visualized to facilitate the development effort of e-Service solutions. Development of a value model is one type of enterprise modeling at very early stages that focus on actors, resources and resource exchanges. 
In this research, we propose a classification schema related to value objects mostly relevant to healthcare industry. Initially BMM [12] is used in analyzing healthcare motivational requirements that leads to classify the value objects. Each value object is represented as a pattern with a description, the motivation for identification and a concrete example illustrating the value object. Further, a set of guidelines is defined in figuring the value objects in an $\mathrm{e}^{3}$-Value model that helps to develop and deploy e-Services effectively.

The value object categorization is described based on a case study from healthcare industry where a patient who needs to admit for a surgery who has already been investigated for a health problem is considered in this paper. Additionally, the proposed classification supports health solution development with the identification of value objects with recurrent use, co-created values and authentication requirements.

The rest of the paper is structured as follows. Section 2 explains research background with relevant standards and related work on how service identification leads to business-IT alignments. Section 3, clearly outlines the proposed Value Object Classification and the set of guidelines to capture the value modeling aspects. Next, Section 4 illustrates how the Value Object Classification and the guidelines are applied to identify services of an enterprise. Finally, Section 5 concludes with discussions.

\section{Related Work and Research Background}

Two main sub-sections are to be introduced here. Firstly the standardizations adopted as the foundations for motivation/goal modeling and healthcare value modeling in our proposed framework. In this context main focus is given on to BMM and $\mathrm{e}^{3}-\mathrm{Value}$ briefly introduce below. Secondly some of the research contributions related to our work in this paper.

\subsection{Service Aware Interoperability Framework (SAIF)}

SAIF is the leading framework that primarily combine recommendations from two global standardization organizations; OMG (Object Management Group-Non-profit organization that introduces standards related to Object Orientated and related IT solution development standards) and HL7 (the internationally recognized organization that introduces messaging standards for Healthcare domain). SAIF focuses to ensure interoperability among healthcare applications [7]. However, SAIF is not completely established with its recommendations and adoptable approach in healthcare solution development. Therefore some research work could be found arguing and proposing different extensions [10]. In this context, our work proposed here could also be considered as possible extensions to SAIF sub-frameworks; Enterprise Conformance and Compliance Framework (ECCF) and Governance Framework (GF).

\subsection{Goal Modeling}

The main composition of a Business Motivation Model (BMM) [12] are ends (such as goals and objectives) and means (such as strategies, tactics, policies, rules) for a 
business environment. BMM shows how goals can be captured and represented using such higher level modeling concepts. Each component of the BMM provides a way to understand the overall business plan and then to facilitate lower level technical realization. Building up components of the BMM consequently may help to identify value objects and the business service processes needed for their exchanges.

Part of such a motivation model developed for the running case introduced here has been illustrated in Fig. 1.

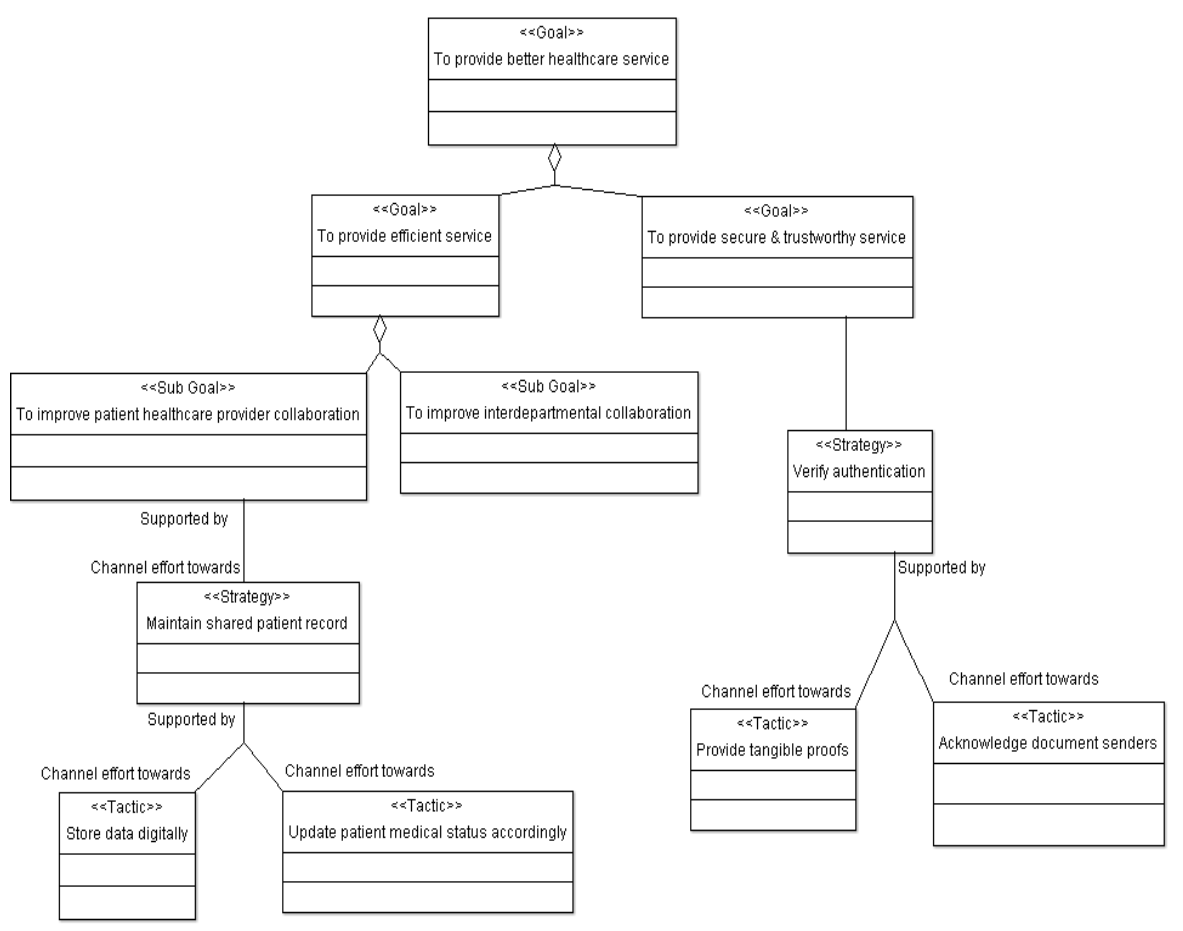

Fig. 1. Motivation model for surgery case

\section{3 $\mathrm{e}^{3}$-Value Constructs}

$\mathrm{e}^{3}$-Value modeling approach is based on an ontology, which has a set of concepts and related notations covering all these major value modeling concepts [3]. It also has methodology to model networked businesses in terms of values and analyzing profitability. Its notation provides graphical representation of businesses by means of value actor, market segment, value object, value port, value interface, value activity, value exchange, etc [4]. A value actor is an economically independent entity who is responsible for profit and loss in business. Market segment represents a set of actors having common economic interests in particular business setup. A commodity that has some economic value for a value actor and involved in exchanges between actors is a value object. A value object can be service, good, money or experience. Value 
ports at value interface of actor's are used to send or receive value objects from/to other actors. Value activities carried out by actors to create/add value to value objects.

\subsection{Motivations/Value Oriented Service Designing}

When representing business environment in value modeling, several methodologies have been proposed by the researchers in order to simplify and to facilitate the process of business solutions development [8], [1], [9], [13].

Recent research works [2], [11] on designing e-Business systems are trying to ensure business-IT alignment by proposing systematic approaches to capture higher level motivational requirements and realizing them on subsequent modeling level of development workflows. Often business models describe what aspects of the organization by focusing on what value is exchanged among actors [11]. It is quite evident the importance of initiating ICT solution development with designing of business models preceded with motivation/goal modeling in order also to guarantee successful business-IT integrations [11], [6].

Mainly values that are exchanging among actors in health industry is considered and analyzed further in our work. Additionally, we focus on identification of eServices in healthcare industry, that facilitate creating values to intended users by analyzing the common characteristics. Our recommendation of healthcare motivation modeling is founded on Business Motivation Model while $\mathrm{e}^{3}$-Value modeling is used as basis for healthcare value modeling in this context. Recent research suggests that how different modeling aspects for identification and development of e-Service of an enterprise. They also suggest a systematic approach that make use of two goal modeling steps and one business modeling step based on $\mathrm{i}^{*}$ and $\mathrm{e}^{3}$-Value modeling methods [1]. Considering goal oriented business modeling researches, we have proposed an alternative approach for identification of Value Objects and thereby initial development of related e-Service for their exchanges among actors.

\section{Value Object Identification in Healthcare Service Sector}

Identification and categorization of different value objects that are being exchanged among different actors in business collaborations are fundamental in designing and development of services. This is the main challenge that e-Service designers are facing and need guidelines and support for business contexts that they engaged in. When considering against typical business collaborations where economic commodities are exchanges, in healthcare industry this situation is very much challenging due to the factors such as involvement of ever increasing numbers of different healthcare service providers and complexity of value objects exchanged among them mostly in the forms of intangible healthcare services.

As a remedy to the situation briefed above, in this work we propose Value Objects Classification for healthcare service industry. Occurrence of following value object patterns have been subjected to this study provoked with the standardization efforts and research work summarized in the above section. 


\subsection{VO Classification}

\section{Intrinsic Value Objects (IVO)}

These are the value objects used in value transfers but the receiving party has no immediate interest other than personal consumption or personal usage with respect to the concerned collaboration context.

Example: The patient fee, a payment is transferred to the admission office from the patient. The received payment will not be transferred in another value transfer in the healthcare collaborations for the case under consideration.

Motivation: IVO have specific usage meant for a single value actor in a particular collaboration.

\section{Recurrent Value Objects (RVO)}

Value objects that are repeatedly used in many different value transfers of a value model. In circumstances receiving party may have some usage or value with RVO and could then again be transferred with or without any value addition to yet another party.

Example: In this case study, same set of medical reports of a patient is transferred among the several actors; from medical laboratory to ward and ward to operation theater, etc. As it is more important to ensure the health state of the patient prior to any medical/surgical treatment related activities, the medical reports are being transferred repeatedly in order to provide necessary patient health state information.

Motivation: RVO are exchanged between many different actors with the same or different value object usages and with or without possible value additions to the received value object.

\section{Co-created Value Objects (CVO)}

These are the value objects that could also be transferred via value transfers but they could also be accesses or/and add value separately or simultaneously by different value actors.

Example: In the case, patient's health records are transferred as patient referral from the admission office to the ward and being updated continuously, same records are accessed and updated by operation theater as well.

Motivation: CVO models the situation where value objects needed to be accessed, continuously updated with possible value additions by many different value actors in a collaboration context.

\section{Affirmative Value Objects (AVO)}

These are kind of auxiliary value objects that in occasions composed with other regular value objects mainly for the authorization and for the verification purposes. These AVO are to be transferred together with other regular value objects.

Example: In the case study for instance certification of issuer in medical reports, certified surgery results and authorized referral statements should be signed by an authorized healthcare service provider. Unless verified with the signature the necessary actions may not be carried out due to policy/regulatory concerns in a particular governing setup. 
Motivation: Authorization, approval and certification of some healthcare value objects are critical. In order to accommodate these requirements we proposed composing regular value objects with AVO through with confirmatory value actor information could be released.

\section{2 $\mathrm{e}^{3}$-Value Modeling Guidelines for the VO Classification}

Set of guidelines can be derived for the afore mentioned Value Object categories as follows and further a relevant framework is provided for the categories to build up the $\mathrm{e}^{3}$-Value model. We have proposed a systematic approach, first to identify Value Objects from these different categories.

\section{Guideline 1}

Identify IVO by looking at goals of value actors' participation in (healthcare/business) collaborations.

Example: Physician's intension of getting fee, patient's intension of getting health service, etc.

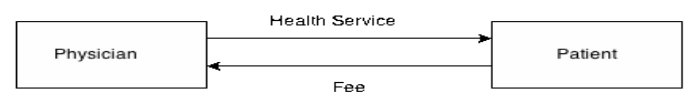

\section{Guideline 2}

Identify RVO that is needed from another party in order to create IVO by a value actor.

Example: Physician in need of a medical report (patient history or pervious treatments, etc)

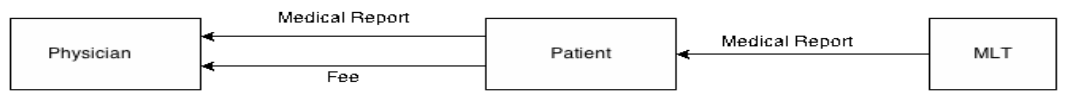

\section{Guideline 3}

Identify CVO that is created in a single or several steps by two or more value actors. This is where multi-party involvement is needed in creation/adding value of objects.

Example: In the operation theater surgeon, antitheists, nursing staff, etc are involved.

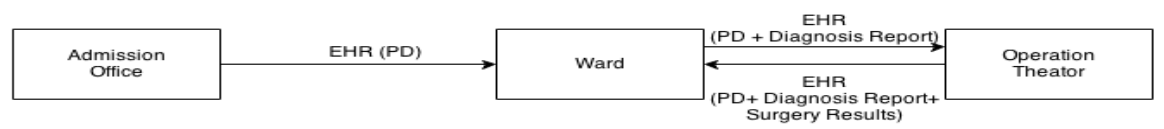

\section{Guideline 4}

Identify AVO that is with or without other type of VO where authority and authorization factors are to be considered as necessary requirement.

Example: Medicine (treatment) of a prescription, its dosage \& usage to be signed and approved by a physician, etc. 


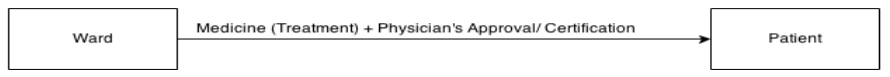

\section{Application of Proposed Classification}

One of the primary requirements in initiating motivation/goal modeling is deciding on a stakeholder's perspective of the concerned business situation. When considering healthcare scenarios it is notable that the ultimate customer is patient and we refer patient's perspective as Primary Perspective in motivation modeling effort. In addition to the Primary Perspective all the other service providers could be considered compassioning healthcare service industry. This could be collectively referred as Healthcare Enterprise Perspective in motivation modeling.

When carefully analyzing healthcare service collaborations, it is evident that there are Governing Collaborations (between Physicians and Patients) that enact and control all the other Auxiliary Collaborations between other supportive healthcare service providers such as Medical Laboratory Technicians, Pharmacists, etc. Therefore, we recommend also to consider these two sub perspectives; Governing Perspective and Auxiliary Perspective also during motivation modeling efforts.

Phase 1: Two perspectives on goal modeling

a.Primary Perspective $\rightarrow \mathrm{HC}$ service concerned goals

b. Enterprise Perspective

- Governing Perspective $\rightarrow$ Overseeing and controlling goals

- Auxiliary Perspective $\rightarrow$ Healthcare Service supportive goals

Phase 2: Goal driven VO identification

a.VOs connected with primary perspective goals

- Primitive VOs

Basically these transfers are IVOs in order to obtain the service.

Ex: Patient to Enterprise $\rightarrow$ Fee (a monitory value)

b. VOs connected with enterprise perspective goals

- Composite VOs

Ex: Enterprise to Patient $\rightarrow$ Healthcare Service (a composite service)

Phase 3: Decomposition of composite value offerings

a.VOs used repeatedly within enterprise with or without value addition

- These transfers are RVOs.

Ex: MLT to Physician $\rightarrow$ Medical Reports

b. VOs involved multi parties who perform co-creations

- These transfers are CVOs.

Ex: Ward to Patient $\rightarrow$ Electronic Health Record (EHR)

c.VOs required authenticity

- These transfers are AVOs.

Ex: Medical Lab to Patient $\rightarrow$ Medical Reports (with MLT's endorsement)

Completed examples have been summarized in Table 1. 
Phase 4: Identification of Actors (supported/intended in the motivation model) who create/consume VOs

Phase 5: Develop e $\mathrm{e}^{3}$-Value model by annotating with above VO categories.

According to the proposed guidelines now it is possible to complete the $\mathrm{e}^{3}$-Value model components for the VOs and actors identified in above phases thus verify the completeness of the final $\mathrm{e}^{3}$-Value model.

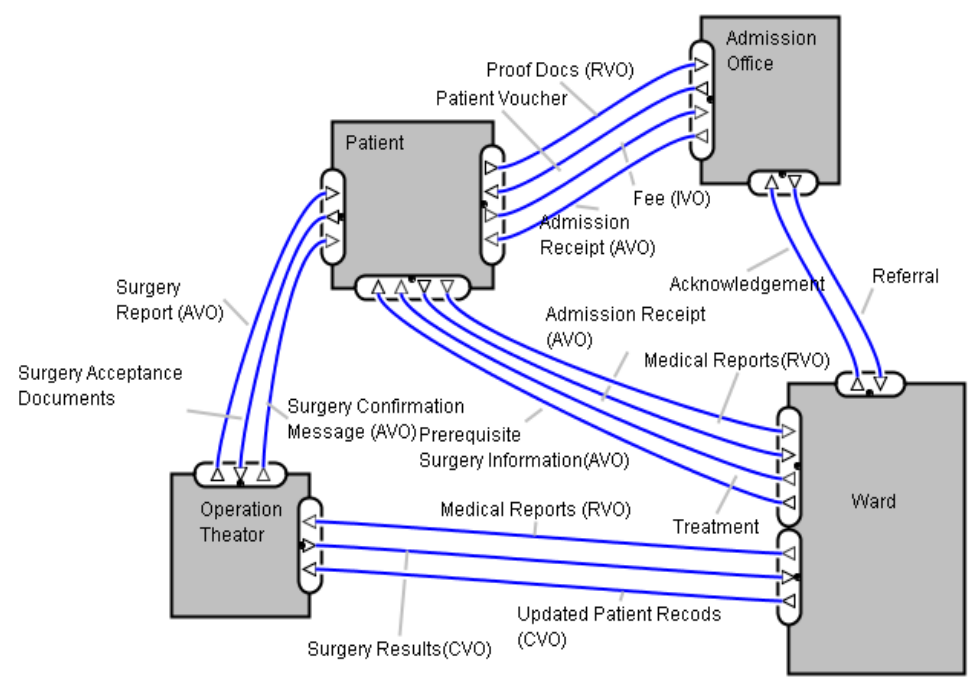

Fig. 2. e3-Value model for surgery case

To illustrate the usage of VO categorization and the guidelines proposed, we developed a simplified value model for the running case according to the above mentioned guidelines and phases. Fig. 2 depicts the extended $\mathrm{e}^{3}$-Value model for the surgery case in health care industry that was verified against the proposed service oriented value object classification.

This work leads easy recognition of VOs transferred among actors in a particular scenario. Then they can be mapped with the proposed VO classification that guides to design $\mathrm{e}^{3}$-Value models by realizing the value actors and the related service activities easily. Table 1 depicts the composition of the value actors, objects and service activities of $\mathrm{e}^{3}$-Value model based on proposed VO categories, for healthcare domain.

In the implementation phase, when developing the IT service solution, several system requirements can be easily realized from the proposed value object classification in line with [1]. Every RVO object is reusable. CVO objects are always shared data that can be accessed concurrently which may demands maintaining information consistencies. Policy, Privacy and Security requirements of value exchanges can also be captured. For instance authenticity of AVO objects can be shown. 
Table 1. VO category for surgery case

\begin{tabular}{|c|c|c|c|c|}
\hline $\begin{array}{l}\text { Value } \\
\text { Object } \\
\text { Category }\end{array}$ & Value Object & Service & Service Provider & $\begin{array}{l}\text { Service } \\
\text { Recipient }\end{array}$ \\
\hline IVO & Fee & Payment & Health Care Institute & Patient \\
\hline \multirow[t]{2}{*}{$\mathrm{RVO}$} & Medical Report* & Medical Report Provision & Medical Lab & Patient \\
\hline & & Medical Report Provision & Patient & Ward \\
\hline \multirow[t]{4}{*}{ CVO } & $\begin{array}{l}\text { Patient Records } \rightarrow \text { Personal } \\
\text { Information }\end{array}$ & Patient Records Provision & Admission Office & Patient \\
\hline & $\begin{array}{l}\text { Patient Records } \rightarrow \text { Personal } \\
\text { Information + Symptoms }\end{array}$ & Patient Records Provision & Ward & Patient \\
\hline & $\begin{array}{l}\text { Patient Records } \rightarrow \text { Personal } \\
\text { Information + Symptoms + } \\
\text { Diagnosis Reports }\end{array}$ & Patient Records Provision & Ward & $\begin{array}{l}\text { Operation } \\
\text { Theater }\end{array}$ \\
\hline & $\begin{array}{l}\text { Patient Records } \rightarrow \text { Personal } \\
\text { Information }+ \text { Symptoms }+ \\
\text { Diagnosis Reports + Surgery } \\
\text { Results }\end{array}$ & Patient Records Provision & Operation Theater & Ward \\
\hline \multirow[t]{2}{*}{ AVO } & Medical Report** & Medical Report Provision & Medical Lab & Patient \\
\hline & Patient Records*** & Patient Records Provision & Ward & Patient \\
\hline
\end{tabular}

* To be produced to the physician, no immediate consumption by patient.

** Medical report together with Medical Laboratory Technician's authorization *** Patient Records together with Consultant's certification

The 05 phases discussed above clearly and precisely shows the applicability of the proposed service oriented value object classification in healthcare domain.

\section{Conclusions}

This work proposes a classification that facilitates identification of different categories of value objects that could exist in healthcare service sector. Since this classification based on goal standardization efforts, our initial experiences show that such taxonomy is capable enough to provide better understanding of services to be developed and deployed by e-Service designers in healthcare industry. The adoption and utilization of the classification could be completed in parallel with business value modeling. Further, it could provide early identification of e-Services of the concerned enterprise application as we have briefly illustrated under the application of the classification. A set of guidelines associated with the value object categories confirms the applicability of proposed classification. This could also help in analyzing the collaborative states and dynamic behavior of different value objects in a service oriented enterprise. This comprehensive set of guidelines assists to build precise $\mathrm{e}^{3}$ Value model based on goals.

Further this can be improved and applied in different specializations and various application areas even within the health industry as well as in other possible domains. We wish to propose a complete framework that combines this classification and set of guidelines along with service rules to different domains that addresses major 
requirements directly influence service process modeling of an organization where it helps to develop and deploy e-Services effectively.

\section{References}

1. Andersson, B., Johannesson, P., Zdravkovic, J.: Aligning goals and services through goal and business modelling. In: Information Systems and e-Business Management, vol. 7(2), pp. 143-169 (March 2009)

2. Baida, Z., Gordijn, J., Sæle, H., Akkermans, H., Morch, A.: An Ontological Approach for Eliciting and Understanding Needs in e-Services. In: Pastor, Ó., Falcão e Cunha, J. (eds.) CAiSE 2005. LNCS, vol. 3520, pp. 400-414. Springer, Heidelberg (2005)

3. Gordijn, J., et al.: The e3-Value methodology (December 17, 2012), http: //e3value.few.vu.nl/

4. Gordijn, J., Akkermans, H.: e3-Value: Design and Evaluation of e-Business Models. IEEE Intelligent Systems 16(4) (2001)

5. Gordijn, J., Kartseva, V., Schildwacht, J., Wieringa, R.J., Akkermans, J.M.: Developing a Domain-Specific Cross-Organizational RE Method. In: Proceedings of The 12th International Conference on Requirements Engineering, RE 2004 (2004)

6. Gordijn, J., Petit, M.: Understanding Business Strategies of Networked Value Constallations Using Value and Goal Modeling. In: Proceedings of 14th IEEE International Conference on Requirements Engineering (2006)

7. HL7 Architecture Board (ed.): HL7 Service Aware Interoperability Framework: Canonical Version, Release 1 (Unique Ballot ID: SAIF CANON R1 I1 2011MAY). HL7 (2011), http://www.hl7.org/ctl.cfm?action=ballots. home

8. Jayaweera, P., Petit, M.: Classifying Business Rules to Guide the Systematic Alignment of a Business Value Model to Business Motivation. In: Proceedings of the Fourth International Workshop on Business/IT Alignment and Interoperability (BUSITAL 2009), Collection CEUR Workshop Proceedings, vol. 456 (2009)

9. Jayaweera, P., Rathnayaka, N.: Rule Framework for Motivation/Service Process Alignment (October 10, 2013), http: / / ict1.tbm. tudelft.nl/vmbo2013/

lib/exe/fetch.php?media=vmbo2013_submission_23.pdf

10. Landgrebe, L., Smith, B.: The HL7 Approach to Semantic Interoperability. In: ICBO: International Conference on Biomedical Ontology, July 28-30 (2011)

11. Martin, H., Paul, J., Erik, P., Jelena, Z.: Value and Goal Driven Design of E-Services. In: IEEE International Conference on E-Business Engineering (ICEBE 2007), pp. 295-303 (2007)

12. Object Management Group, Inc. Business Motivation Model, BMM (December17, 2012), http: / / www . omg . org/spec / BMM/

13. Raadt van der, B., Gordijn, J., Yu, E.: Exploring web services ideas from a business value perspective. In: Atlee, J., Roland, C. (eds.) Proceedings of the 2005 13th IEEE International Conference on Requirements Engineering (RE 2005), pp. 53-62. IEEE Computer Society, Los Alamitos (2005)

14. Semantics Of Business Vocabulary And Business Rules (SBVR), Version 1.0. (January 03, 2014), http: / /www.omg.org/spec/SBVR/1.0/ 